Pacific Journal of Mathematics

COHOMOLOGY OF CYCLIC GROUPS OF PRIME SQUARE 


\title{
COHOMOLOGY OF CYCLIC GROUPS OF PRIME SQUARE ORDER
}

\author{
J. T. PARR
}

Let $G$ be a cyclic group of order $p^{2}, p$ a prime, and let $U$ be its unique proper subgroup. If $A$ is any $G$-module, then the four cohomology groups

$$
H^{0}(G, A) \quad H^{1}(G, A) \quad H^{0}(U, A) \quad H^{1}(U, A)
$$

determine all the cohomology groups of $A$ with respect to $G$ and to $U$. This article determines what values this ordered set of four groups takes on as $A$ runs through all finitely generated $G$-modules.

Reduction. Let $G$ be any finite group. A finitely generated $G$ module $M$ is quotient of a finitely generated $G$-free module $L$. The kernel $K$ is $Z$-free, and since the cohomology of $L$ is zero with respect to all subgroups of $G, K$ is a dimension shift of $M$. The standard dimension shifting module $P=Z G /\left(S_{G}\right)$ is $Z$-free, so $K \otimes P$ is a $Z$-free $G$-module having the same cohomology as $M$ with respect to all subgroups of $G$.

Proposition 1. If $G$ is any finite $p$-group and $M$ any $Z$-free $G$ module, the cohomology of $M$ is that of $R \otimes M$ where $R$ is the ring of $p$-adic integers.

Proof. Because $M$ is $Z$-free, $0 \rightarrow M \rightarrow R \otimes M \rightarrow R / Z \otimes M \rightarrow 0$ is a $G$-exact sequence. $R / Z \otimes M$ is divisible and $p$-torsion free, so its cohomology is zero, and $M \rightarrow R \otimes M$ induces isomorphism on all cohomology groups.

If $M$ is $Z$-free and finitely generated, $R \otimes M$ is an $R$-torsion free, finitely generated $R G$-module. So we see that if $G$ is any finite $p$-group, every finitely generated $G$-module has the same cohomology as a finitely generated, $R$-torsion free $R G$-module.

2. Exact sequences. Let $G$ be generated by an element $g$ of order $p^{2}$ and let $U$ be its subgroup of order $p$. Heller and Reiner [2] have determined all indecomposable finitely generated $R$-torsion free $R G$-modules:

(a) $R$ with trivial action

(b) $B=R(\omega)$, $\omega$ a primitive $p$ th root of $1, g \omega^{j}=\omega^{j+1}$

( c ) $C=R(\theta), \theta$ a primitive $p^{2}$ th root of $1, g \theta^{j}=\theta^{j+1}$

Received December 27, 1963. 
(d) $E=R H, H$ a cyclic group of order $p$ generated by $h$, $g h^{j}=g h^{j+1}$

(e)-(i) a module $M$ such that there exists an exact sequence

( e ) $0 \rightarrow R \rightarrow M \rightarrow C \rightarrow 0$

(f) $0 \rightarrow E \rightarrow M \rightarrow C \rightarrow 0$

(g) $\quad 0 \rightarrow B \rightarrow M \rightarrow C \rightarrow 0$

(h) $\quad 0 \rightarrow R \oplus E \rightarrow M \rightarrow C \rightarrow 0$

(i) $0 \rightarrow R \oplus B \rightarrow M \rightarrow C \rightarrow 0$

We compute the cohomology of the modules in (a)-(d) directly, and find their sets of four groups to be
(a) $Z_{p^{2}}$
0
$Z_{p} \quad 0$
(b) 0
$Z_{p} \quad(p-1) Z_{p}$
(c) 0
$Z_{p}$
(d) $Z_{p}$
0
0
0
$p Z_{p}$
$p Z_{p}$
0

The exact cohomology sequences arising from the exact sequences (e)-(i) restrict the cohomology possibilities to

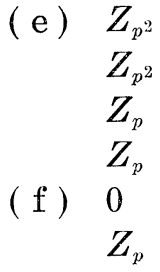
$Z_{p}$
$Z_{p}$
$Z_{p}$
0
$Z_{p}$
$p Z_{p}$
0
0
0
0
$Z_{p}$
$n Z_{p}$
$n=0, \cdots, p$
( g ) $\begin{aligned} & 0 \\ & 0\end{aligned}$
$\begin{array}{lll}2 Z_{p} & n Z_{p} & (n+1) Z_{p} \\ Z_{p^{2}} & n Z_{p} & (n+1) Z_{p}\end{array}$
$n=0, \cdots, p-1$
( h ) $\begin{aligned} & Z_{p^{2}} \\ & \\ & 2 Z_{p} \\ & \\ & Z_{p^{2}}+Z_{p}\end{aligned}$
0
$(n+1) Z_{p}$
$n Z_{p}$
$0 \quad(n+1) Z_{p}$
$Z_{p}$
$(n+1) Z_{p}$$$
n=0, \cdots, p
$$
( i ) $\begin{aligned} & Z_{p^{2}} \\ & Z_{p^{2}} \\ & \\ & Z_{p}\end{aligned}$
$Z_{p^{2}}$
$2 Z_{p}$
$Z_{p}$
$n Z_{p}$
$n Z_{p}$
$(p-1) Z_{p}$
$p Z_{p}$
$(p-1) Z_{p}$
$n Z_{p}$

$\begin{array}{lr}Z_{p^{2}} & Z_{p} \\ Z_{p^{2}} & 2 Z_{p} \\ Z_{p} & Z_{p}\end{array}$

$$
n=0, \cdots, p
$$

In $\S 4$ we shall determine which of these combinations actually occur.

3. Enlargements. An $R$-enlargement of $C$ by $A$ is an $R$-split $R G$-exact sequence $0 \rightarrow A \rightarrow M \rightarrow C \rightarrow 0$ [1]. Two enlargements involving $M$ and $M^{\prime}$ are equivalent if there exists an $R G$-homomorphism $u: M \rightarrow M^{\prime}$ such that 


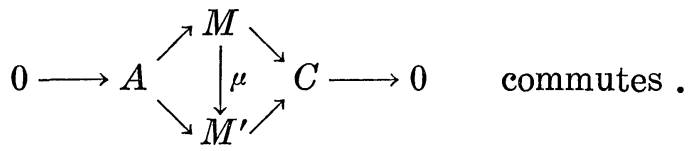

The $R$-split exact sequence gives $M$ the $R$-structure of $A \oplus C$. The first summand is determined by the sequence, but the second is not; choose any one of the poss ble $R$-submodules for the second summand. Because the sequence is a $G$-sequence, $g(a, 0)=(g a, 0)$ and the second component of $g(0, c)$ is $g c$. Denote the first component of $g(0, c)$ by $f(c) ; g(0, c)=(f(c), g c)$. So $f$ is a function from $C$ into $A$, and is an $R$-homomorphism because $g$ is an $R$-homomorphism. The equation $g^{p^{2}}(0, c)=\left(\left(N_{G} f\right)(c), c\right)=(0, c)$ gives us that $f$ is a - 1-cocycle of the $G$-module $\operatorname{Hom}_{R}(C, A)$ where $G$ acts by $(g f)(c)=g f\left(g^{-1} c\right)$. Clearly, every - 1-cocycle defines an action by $G$ on $A \oplus C$ which makes an $R$-enlargement of $0 \rightarrow A \rightarrow A \oplus C \rightarrow C \rightarrow 0$. If two -1 -cocycles $f_{1}$ and $f_{2}$ differ by a coboundary, $f_{1}-f_{2}=(g-1) f_{3}$, then

$$
u(\alpha, c)=\left(\alpha+\left[(1-g) f_{3}\right]\left(g^{-1} c\right), c\right)
$$

defines an $R G$-isomorphism $u$ of $A \oplus C$ with $G$-module structure given by $f_{1}$ onto $A \oplus C$ with $G$-module structure given by $f_{2}$; the $R G$-modules corresponding to $f_{2}$ and $f_{1}$ are isomorphic. So to investigate all enlargement modules $M$ of $C$ by $A$ we need only look at those corresponding to a set of representative cocycles of $H^{-1}\left(G, \mathbb{H}_{1} m_{R}(C, A)\right)$.

Since the modules $R, B, C$, and $E$ are $R$-free, the exact sequences (e)-(i) are $R$-split, and $M$ is an enlargement in each case of $C$ by another module.

For the application of this section, we shall need the following propositions.

Proposition 2. If $A$ is an $R G$-module on which $U$ acts trivially, then $N_{G} \operatorname{Hom}_{R}(C, A)=0$.

Proof. Let $f \in \operatorname{Hom}_{R}(C, A)$. We easily compute that $\left(N_{G} f\right)\left(\theta^{j}\right)=$ $g^{j}\left(N_{G} f\right)(1)$, and using the facts that $\theta$ satisfies

$$
x^{p(p-1)}+x^{p(p-2)}+\cdots+x^{p}+1=0
$$

and that $g^{p}$ acts trivially on $A$, we find by writing it out that $\left(N_{G} f\right)(1)=0$, which then implies that $N_{G} f=0$.

Abbreviate $p(p-1)=m$. Since $C$ is the $R$-direct sum of the $R$-submodules generated by $\theta^{i}, i=0,1, \cdots, m-1$, then $\operatorname{Hom}_{R}(C, A)$ is the direct sum of subgroups $F_{i}$, where $F_{i}$ is the set of all $R$-homomorphisms from $C$ to $A$ which have value zero for all $\theta^{j}$ except possibly for $j=i$. 
Proposition 3. If $A$ is any $R G$-module, every element of $\mathrm{Hom}_{R^{-}}$ $(C, A)$ is equivalent mod the -1 -coboundary group $(g-1) \operatorname{Hom}_{R}(C, A)$ to some element of $F_{m-1}$.

Proof. If $f \in F_{0}$, then $g^{-1} f \in F_{m-1}$, and $g^{-1} f-f=\left(g^{-1}-1\right) f=$ $(g-1)\left(g^{p^{2}-2}+\cdots+g+1\right) f$. If $f \in F_{i}$, then $g f \in F_{i+1}+F_{0}$ differs from $f$ by $(g-1) f$. The proof succeeds by repeated application of these cases to the $F_{i}$-components of an arbitrary $f$.

Corollary. If $M$ is one of the modules described in (e)-(i), $M$ is an enlargement module of $C$ by $A(A=R, B, E, R \oplus B, R \oplus E)$ corresponding to an element of $F_{m-1}$.

Because we are concerned only with indecomposable modules, the following proposition will spare us some unnecessary computations later on.

Proposition 4. Let $M$ be an enlargement module of $C$ by $A \oplus D$ corresponding to $f \in \operatorname{Hom}_{R}(C, A \oplus D) \cong \operatorname{Hom}_{R}(C, A) \oplus \operatorname{Hom}_{R}(C, D)$, and let $f=f_{1}+f_{2}$ be the corresponding decomposition of $f$. Then if either $f_{1}$ or $f_{2}$ represents a $G$-split enlargement of $C$ by $A$ or $D, M$ is decomposable as a $G$-module.

Proof. Suppose $f_{1}$ represents an $R G$-split enlargement of $C$ by $A$. Let $N$ be $A \oplus C$ with action of $C$ defined by $f_{1}$. Since the enlargement splits there is an $R G$-homomorphism $w: N \rightarrow A$ such that $A \rightarrow$ $N \rightarrow A$ is the identity of $A$. Let $u$ be the restriction of $w$ to the given copy of $C$ in $\mathrm{N}$. That $w$ is an $R G$-homomorphism right inverse to the inclusion of $A$ in $N$ requires that $g u(c)=f_{1}(c)+u(g c)$.

Let $M$ be $A \oplus D \oplus C$ with action of $G$ defined by $f$. Then $v(a+d+c)=a+u(c)$ defines an $R G$-homomorphism right inverse to the inclusion of $A$ in $M$, so $M$ is decomposable as an $R G$-module.

4. Computations. In this section we determine which of the possibilities for the cohomology of (e)-(i) actually occur.

Proposition 5. Let $A$ be an $R G$-module left fixed by $U$, and let $M$ be an enlargement module of $C$ by $A$ corresponding to $f \in F_{m-1}$. Then

i) $H^{0}(G, M)=A^{\sigma} /\left(N_{G} A+N_{\text {alU }} f\left(\theta^{m-1}\right)\right)$

ii) $H^{0}(U, M)$ is isomorphic to the quotient of $A / N_{U} A$ with respect to the cyclic $G / U$-submodule generated by the class of $f\left(\theta^{m-1}\right)$.

Proof. $M^{\theta}$ is just the copy of $A^{\theta}$ canonically (by the given exact sequence) contained in $M, M^{U}$ the copy of $A^{U}$. Since $A$ is a submodule, 
the norms of elements of the copy of $A$ are the images of the norms in $A$. Computation shows

$$
\begin{aligned}
& N_{G}\left(0, \theta^{i}\right)=N_{G}(0,1)=\left(N_{G / U} f\left(\theta^{m-1}\right), 0\right) \\
& N_{U}\left(0, \theta^{i}\right)=g^{i} N_{U}(0,1)=g^{i}\left(f\left(\theta^{m-1}\right), 0\right)
\end{aligned}
$$

whence the result.

We are now able to settle case (e).

(e) $M$ is an enlargement module of $C$ by $R$. By Proposition 5 , $H^{0}(G, M)$ is $Z_{p^{2}}$ if $f\left(\theta^{m-1}\right)$ is a multiple of $p$ and $Z_{p}$ if not; and $H^{0}(U, M)$ is $Z_{p}$ if $f\left(\theta^{m-1}\right)$ is a multiple of $p$ and 0 if not. This, together with the information in Section 3, shows that the only cohomology this module $M$ might have is

$\begin{array}{ccccc} & Z_{p^{2}} & Z_{p} & Z_{p} & p Z_{p} \\ \text { or } & Z_{p} & 0 & 0 & (p-1) Z_{p} .\end{array}$

For the remaining cases, we shall need one more proposition.

Proposition 6. Let $H$ be a group of order $p$ generated by $h$. Let $A$ be a cyclic $Z_{p} H$-module of $Z_{p}$-dimension $n$. Then

(i) $(h-1)^{j} A$ has dimension $n-j, j=0, \cdots, n$.

(ii) $a$ is a generator for $A$ if and only if $a \notin(h-1) A$.

(iii) $a$ is a generator for $A$ if and only if $(h-1)^{n-1} a$ is nonzero.

Proof. (i) We have a properly descending chain

$$
A \supset(h-1) A \supset \cdots \supset(h-1)^{n-1} A \supset(h-1)^{n} A=0
$$

of $Z_{p}$-spaces, and we can see by counting that the dimension of $(h-1)^{j} A$ is $n-j$.

(ii) The above chain exhibits all submodules of $A$.

(iii) If a generates $A,(h-1)^{n-1} a$ generates $(h-1)^{n-1} A$, which is not zero. If not, $a \in(h-1) A$, so $(h-1)^{n-1} a=0$.

(f) $M$ is an enlargement module of $C$ by $E, E / p E=\bar{E}$ is a cyclic $Z_{p}(G / U)$-module of $Z_{p}$-dimension $p$. Let $M$ be represented by $f \in F_{m-1}$, and $f\left(\theta^{m-1}\right)=e$. By Proposition $5, H^{0}(G, M)$ is the quotient of $H^{0}(G, E)$ by the subgroup generated by $N_{G / U} \bar{e}=(\bar{g}-1)^{p-1} \bar{e}$, hence zero if $N_{G / U} \bar{e}$ is not zero, $Z_{p}$ if it is. Using proposition 6 iii, we see

$$
\begin{aligned}
H^{0}(G, M) & \cong 0 \text { if } \bar{e} \text { generates } \bar{E} \text { over } Z_{p}(G / U) \\
& \cong Z_{p} \text { if not } .
\end{aligned}
$$

$H^{0}(U, M)$ is the quotient of $H^{0}(U, E) \cong \bar{E}$ by the $Z_{p}(G / U)$ submodule generated by $\bar{e}$. Let $n$ be the largest integer with $\bar{e} \in(g-1)^{n} \bar{E}$. By Proposition 6 ii then, $\bar{e}$ generates $(g-1)^{n} \bar{E}$, which is of dimension $p-n$, so the quotient has dimension $n$. The coho- 
mology of $M$ is

$$
\begin{array}{cccccc}
0 & 0 & 0 & 0 & \text { if } & n=0 \\
Z_{p} & Z_{p} & n Z_{p} & n Z_{p} & \text { if } & n=1, \cdots, p .
\end{array}
$$

(g) $\quad M$ is an enlargement module of $C$ by $B . \quad N_{G} M \subset M^{\theta}=B^{\theta}=$ 0 . So $H^{0}(G, M)=0$ and $H^{1}(G, M) \cong H^{-1}(G, M)$ is the quotient of $M$ modulo $(g-1) M$. Let $M$ correspond to $f \in F_{m-1}$ and denote $f\left(\theta^{m-1}\right)=b$.

Case 1. $b \in(g-1) B$. Then $H^{1}(G, M) \cong 2 Z_{p}$

Case 2. $b \notin(g-1) B$. Then $H^{1}(G, M) \cong Z_{p^{2}}$.

By Proposition 6 again,

$$
\begin{aligned}
H^{1}(G, M) & \cong 2 Z_{p} \text { if } \bar{b} \text { does not generate } B / p B \\
& \cong Z_{p^{2}} \text { if it does }
\end{aligned}
$$

Similarly as in (f), if $n$ is the greatest integer with $\bar{b} \in(\bar{g}-1)^{n}(B / p B)$, then $H^{0}(U, B) \cong n Z_{p}$. The cohomology is thus

$\begin{array}{rrrrll}0 & Z_{p^{2}} & 0 & Z_{p} & \text { if } & n=0 \\ 0 & 2 Z_{p} & n Z_{p} & (n+1) Z_{p} & \text { if } & n=1, \cdots, p-1 .\end{array}$

(h) $M$ is an enlargement module of $C$ by $R \oplus E$. Let $M$ correspond to $f \in F_{m-1}$ and write $f\left(\theta^{m-1}\right)=r+e, r \in R, e \in E$. We may assume $r$ is not divisible by $p$, because if it were, $M$ would be decomposable (Proposition 4).

Computation based on Proposition 5 shows

$$
\begin{aligned}
H^{\circ}(G, M) & \cong 2 Z_{p} \text { if } N_{G / U} e \text { is divisible by } p \\
& \cong Z_{p^{2}} \text { if not, }
\end{aligned}
$$

and that

$$
\begin{array}{rlrl}
H^{\circ}(U, M) & \cong(n+1) Z_{p} & \text { if } & n=0, \cdots, p-1 \\
\cong p Z_{p} & \text { if } & n=p
\end{array}
$$

where $n$ is the largest integer with $\bar{e} \in(g-1)^{n} \bar{E}$. So the cohomology of $M$ may be

$$
\begin{array}{rrrrl}
Z_{p^{2}} & 0 & Z_{p} & 0 & \text { or } \\
2 Z_{p} & 0 & (n+1) Z_{p} & n Z_{p} & n=1, \cdots, p-1 .
\end{array}
$$

(i) $M$ is an enlargement module of $C$ by $R \oplus B$. Let $f \in F_{m-1}$ represent the enlargement and write $f\left(\theta^{m-1}\right)=r+b, \quad \mathrm{r} \in R, \quad b \in B$. Again we may assume $r$ is not divisible by $p$.

$H^{0}(G, M) \cong Z_{p}$ by Proposition 5 . 
Let $j$ be the largest integer with $\bar{b} \in(g-1)^{j} \bar{B}$.

$$
\begin{aligned}
H^{0}(U, M)=(j+1) Z_{p} & \text { if } \quad j=0, \cdots, p-2 \\
=(p-1) Z_{p} & \text { if } \quad j=p-1 .
\end{aligned}
$$

So the cohomology of $M$ is

$$
Z_{p} \quad Z_{p} \quad n Z_{p} \quad n Z_{p} \quad n=1, \cdots, p-1 .
$$

5. Summary. If $M$ is any finitely generated $G$-module, then the cohomology of $M$ is the direct sum of a finite number of the following:

$\begin{array}{lccccc} & H^{0}(G, A) & H^{1}(G, A) & H^{0}(U, A) & H^{1}(U, A) \\ \text { 1. } & Z_{p^{2}} & 0 & Z_{p} & 0 & \\ \text { 2. } & 0 & Z_{p^{2}} & 0 & Z_{p} & \\ \text { 3. } & Z_{p} & 0 & p Z_{p} & 0 & \\ \text { 4. } & 0 & Z_{p} & 0 & p Z_{p} & \\ \text { 5. } & Z_{p} & 0 & 0 & (p-1) Z_{p} & \\ 6 . & 0 & Z_{p} & (p-1) Z_{p} & 0 & \\ \text { 7. } & Z_{p} & Z_{p} & n Z_{p} & n Z_{p} & n=1, \cdots, p \\ \text { 8. } & 2 Z_{p} & 0 & (n+1) Z_{p} & n Z_{p} & n=1, \cdots, p-1 \\ \text { 9. } & 0 & 2 Z_{p} & n Z_{p} & (n+1) Z_{p} & n=1, \cdots, p-1\end{array}$

Given any direct sum of finitely many of the above, there is a finitely generated $G$-module with that cohomology.

\section{BIBLIOGRAPHY}

1. Samuel Eilenberg, Topological methods in abstract algebra, Bull. Amer. Math. Soc. 55 (1949), 3-35.

2. A. Heller and I. Reiner, Representations of cyclic groups in rings of integers $I$. Ann. of Math. 76 (1962), 73-92. 



\title{
PACIFIC JOURNAL OF MATHEMATICS
}

\author{
EDITORS
}

\section{H. SAMELSON}

Stanford University

Stanford, California

R. M. Blumenthal

University of Washington

Seattle, Washington 98105

\section{*J. DugundJI}

University of Southern California Los Angeles, California 90007

\section{RichaRd ARENS}

University of California

Los Angeles, California 90024

\section{ASSOCIATE EDITORS}
E. F. BECKENBACH
B. H. NEUManN
F. WOLF
K. YOSIDA

\section{SUPPORTING INSTITUTIONS}

\author{
UNIVERSITY OF BRITISH COLUMBIA \\ CALIFORNIA INSTITUTE OF TECHNOLOGY \\ UNIVERSITY OF CALIFORNIA \\ MONTANA STATE UNIVERSITY \\ UNIVERSITY OF NEVADA \\ NEW MEXICO STATE UNIVERSITY \\ OREGON STATE UNIVERSITY \\ UNIVERSITY OF OREGON \\ OSAKA UNIVERSITY \\ UNIVERSITY OF SOUTHERN CALIFORNIA
}

\author{
STANFORD UNIVERSITY \\ UNIVERSITY OF TOKYO \\ UNIVERSITY OF UTAH \\ WASHINGTON STATE UNIVERSITY \\ UNIVERSITY OF WASHINGTON \\ AMERICAN MATHEMATICAL SOCIETY \\ CHEVRON RESEARCH CORPORATION \\ TRW SYSTEMS \\ NAVAL ORDNANCE TEST STATION
}

\footnotetext{
Mathematical papers intended for publication in the Pacific Journal of Mathematics should be typewritten (double spaced). The first paragraph or two must be capable of being used separately as a synopsis of the entire paper. It should not contain references to the bibliography. Manuscripts may be sent to any one of the four editors. All other communications to the editors should be addressed to the managing editor, Richard Arens at the University of California, Los Angeles, California 90024 .

50 reprints per author of each article are furnished free of charge; additional copies may be obtained at cost in multiples of 50 .
}

The Pacific Journal of Mathematics is published monthly. Effective with Volume 16 the price per volume (3 numbers) is $\$ 8.00$; single issues, $\$ 3.00$. Special price for current issues to individual faculty members of supporting institutions and to individual members of the American Mathematical Society: $\$ 4.00$ per volume; single issues $\$ 1.50$. Back numbers are available.

Subscriptions, orders for back numbers, and changes of address should be sent to Pacific Journal of Mathematics, 103 Highland Boulevard, Berkeley 8, California.

Printed at Kokusai Bunken Insatsusha (International Academic Printing Co., Ltd.), No. 6, 2-chome, Fujimi-cho, Chiyoda-ku, Tokyo, Japan.

PUBLISHED BY PACIFIC JOURNAL OF MATHEMATICS, A NON-PROFIT CORPORATION

The Supporting Institutions listed above contribute to the cost of publication of this Journal, but they are not owners or publishers and have no responsibility for its content or policies.

* Paul A. White, Acting Editor until J. Dugundji returns. 


\section{Pacific Journal of Mathematics}

\section{Vol. 17, No. $3 \quad$ March, 1966}

Tsuyoshi Andô, Contractive projections in $L_{p}$ spaces ............... 391

Robert F. Brown, On a homotopy converse to the Lefschetz fixed point theorem ............................................... 407

Richard Albert Cleveland and Sandra Cleveland, On the multiplicative extension property................................ 413

Harold H. Johnson, An algebraic approach to exterior differential systems..................................... 423

Alan Cecil Lazer, The behavior of solutions of the differential equation

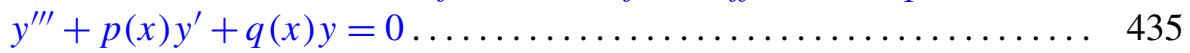

Judy Parr, Cohomology of cyclic groups of prime square order ......... 467

Donald Steven Passman, Groups whose irreducible representations have

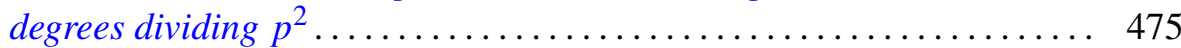

Ralph Tyrrell Rockafellar, Characterization of the subdifferentials of convex functions ........................................... 497

Donald Erik Sarason, Invariant subspaces and unstarred operator algebras...................................... 511

Donald Erik Sarason, Weak-star generators of $H^{\infty} \ldots \ldots \ldots \ldots \ldots \ldots . \ldots . \ldots$

Boris M. Schein, Homomorphisms and subdirect decompositions of semi-groups

Daniel Francis Shea, Jr., Functions analytic in a finite disk and having asymptotically prescribed characteristic

Zvi Ziegler, Generalized convexity cones 